\title{
Simulation of a 2D Magnetic Drug Targeting Model
}

\author{
MS. AGGOUNE, N. AGGOUNE, N. BENSEDIRA, A.BOUHANTALA and A. BELKBIR
}

\begin{abstract}
One of the major drawbacks of chemotherapy in cancer treatment of various human organs is the fact that the used anticancer therapeutics (medication) inevitably attack both healthy tissue and tumors. In order to guide the drug to a specific location, the optimal technique used within the human body is the magnetic drug targeting (MDT) due to its non-invasive character and its high targeting efficiency. In this method, magnetic carrier particles loaded with drug molecules are injected into the microvasculature and attracted towards the targeted region in the body by a permanent magnet. In this work a mathematical model is developed for measuring the trajectories of the nanoparticles, their direction and the flow speed. The mixed blood flow enters a $1 \mathrm{~cm}$ large vessel where blood is being pumped by the heart pulses. The simulation investigates whether the flow will follow a vessel located closer to the entrance of the fluid flow away from the magnet or whether the magnetic field will pull the nanoparticles towards the area of interest beneath the magnet. The model couples a magneto static model to a hydrodynamic problem taking into account the dominant magnetic force. It is assumed that the effect of the electric field is negligible. The results obtained from the numerical solution showed that the fluid flow is appreciably influenced by the applied magnetic field and that the ferro fluid is attracted to the zone where the magnet is located whereas the normal blood can reach all tissues. (Abstract)
\end{abstract}

Keywords-MDT, magnetic drug targeting, nanoparticles, magnetic field, cancer, numerical simulation.

\section{Introduction}

The delivery of anticancer agents to the specific target sites with minimum side effects is an important challenge in chemo, radio and gene-therapy [1]. During chemotherapy, typically less than 0.1 to $1 \%$ of the drugs are taken up by tumor cells, with the remaining 99\% going into healthy tissue [2] .Magnetic Drug Targeting (MDT) is one of the promising methods for effective targeting and delivery of drugs to a specific location by means of an external magnetic field [1].

In this method, magnetic carrier nanoparticles loaded with drug molecules are injected into the blood flow as a dilution and attracted towards the targeted region in the body with help of a local magnetic field [3]. With this technique the efficiency of drug absorption increases and the other cells of body will not be affected [4].

Mohammed-Salah Aggoune, N. Aggoune, N. Bensedira, A.BouhantalA and A. Belkbir

LEB Laboratory, University of Batna 2

Algeria

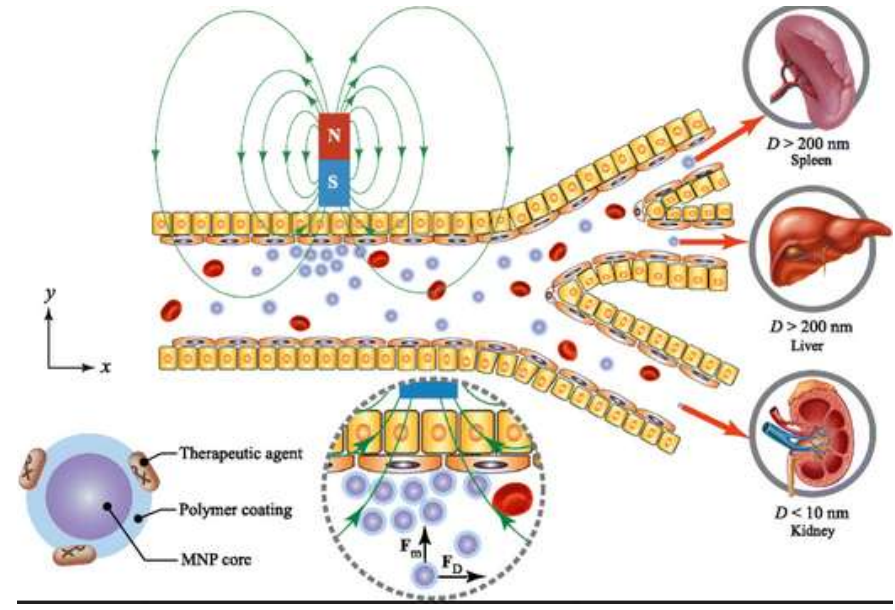

Figure 1. Modeled image of a MDT in various tissues [3].

Drug-loaded carrier is typically composed of a magnetic core and a biocompatible coating material. The magnetic core was made from different materials such as $\mathrm{Fe} 3 \mathrm{O} 4, \mathrm{Fe} 2 \mathrm{O} 3$, or $\mathrm{Fe}$ [19] .The magnetite ( $\mathrm{Fe} 3 \mathrm{O} 4)$ nanoparticles are generally used in targeted drug delivery systems because of their biocompatibility and large magnetization [4]. The biocompatible coating materials are Au, PEG, or $\mathrm{SiO} 2$. Based on the biokinetics of particles, a drug carrier ranging from 10 $200 \mathrm{~nm}$ in diameter is optimal for in vivo delivery, as the small particles $(\mathrm{D}<10 \mathrm{~nm})$ escape by renal clearance and the large ones (D>200 nm) are sequestered by the reticuloendothelial system of the spleen and liver [3].

Ferrofluids are suspensions of single domain magnetite particles with average diameters of approximately $10 \mathrm{~nm}$ stabilized by surfactants in carrier liquids [5]. MDT is growing due to speedy progress in the growth of functionalized magnetic nanoparticles, which are used for chemo, radio, and gene-therapy at a tumour site[1]. It is also shown by various studies that MDT is relatively safe and effective method for targeting drugs to a localized region [1, 2, 3-4]. Previous work on magnetic particles transport in the vasculature for MDT is summarized in many review papers [6-9].

In the first human trials of magnetic drug targeting the chemotherapy drug epidoxorubicin was attached to $100 \mathrm{~nm}$ diameter bio-compatible iron-core particles, these particles were administered systemically, and an external magnet was used to concentrate the therapy to inoperable but shallow tumors [10]. In these human safety trials, blood sample it showed that the magnet removed about half of the particles from blood circulation and collected them to the vicinity of the tumor [10-12].

In the present work, a mathematical model is developed to describe the trajectories of the ferrofluid. For this purpose, a 2D model is suggested, consisting of a blood vessel $1 \mathrm{~cm}$ in 
width entering a mass tissue which then divides into two branches. The first branch feeds a healthy tissue and the remaining branch feeds a hypothetical tumor located at the end of the canal (figure 2). An external magnet and the surrounding environment are also represented. The magnetic carrier nanoparticles loaded with drug molecules are injected into the blood which flows from left towards both branches. The magnet is placed above the skin tissue to provide a permanent magnetic interaction with the carried ferrofluids in the blood vessel.

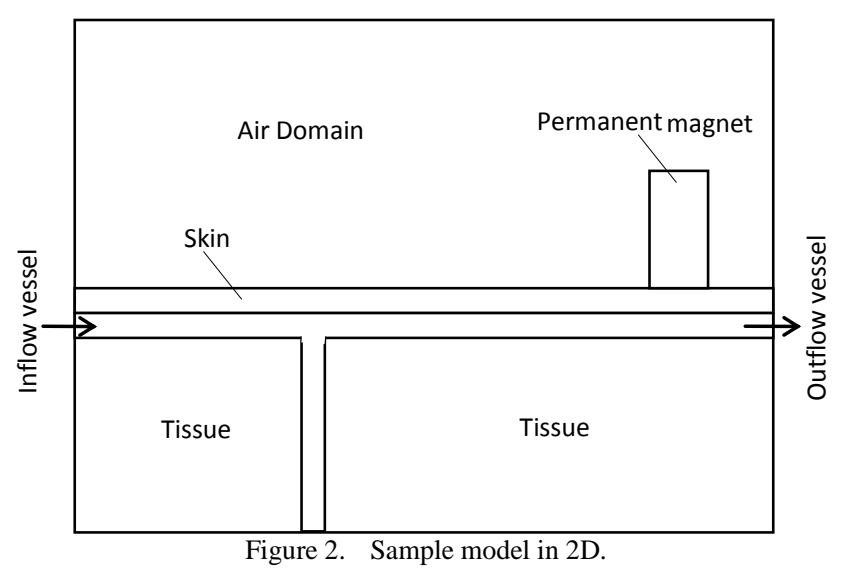

The purpose of this work is to investigate, by simulation, if we would be able to magnetically direct and attract the magnetized nanoparticles even when closer vessels to the main blood entrance are present prior to the magnet location and, hence, attract and guide them towards the desired site.

\section{The mathematical model}

The MDT modeling addresses many coupled problems of which, the magnetic field in its static state, the hydrodynamic, the thermal and the structural mechanics are the most important. To investigate the effect of an applied magnetic field on the velocity and the direction of blood carrying the super-paramagnetic nanoparticles coated with medication, the simulation was carried out to solve coupled Maxwell and Navier-Stokes equations.

\section{A. Magneto static Modeling:}

When solving a magnetic or an electrical problem, it is often useful to use the appropriate potentials instead of the fields. The PDE (Partial Differential Equations) obtained using Maxwell's equations in terms of the electric scalar potential $\mathrm{V}$ and the magnetic vector potential A are adopted in the present work.

According to Maxwell-Ampere's law:

$$
\boldsymbol{\nabla} \times \boldsymbol{H}=\boldsymbol{J}
$$

where the magnetic field $\mathrm{H}(\mathrm{A} / \mathrm{m})$ and the total current density $J(A / m 2)$ could be expressed respectively in terms of the magnetic flux density $B(T)$, the magnetization vector $M$ $(\mathrm{A} / \mathrm{m})$, the electrical field $\mathrm{E}(\mathrm{V} / \mathrm{m})$ and the velocity $\mathrm{u}$ of the moving body. The relevant laws and constitutive relations are listed below:

$$
\begin{gathered}
\boldsymbol{J}=\sigma \boldsymbol{E} \\
\mathbf{E}=\mathbf{u} \times \mathbf{B}-\left(\frac{\partial \mathbf{A}}{\partial t}+\nabla \cdot \mathbf{V}\right) \\
\boldsymbol{\nabla} \cdot \boldsymbol{B}=\mathbf{0} \\
\boldsymbol{B}=\boldsymbol{\nabla} \times \boldsymbol{A} \text { with } \boldsymbol{\nabla} \cdot \mathbf{A}=\mathbf{0}
\end{gathered}
$$

where: (2) is the Ohm's Law, (3) is Total electrical field, (4) is Magnetic flux conservation and (5) is Magnetic vector potential

Magnetic f lux density expression in different media:

$$
\begin{aligned}
\boldsymbol{B} & =\mu_{0} \mu_{r} \mathbf{H}+\mathbf{B}_{\mathrm{r}} \\
\boldsymbol{B} & =\mu_{0}(\boldsymbol{H}+\boldsymbol{M}) \\
\boldsymbol{B} & =\mu_{0} \boldsymbol{H}
\end{aligned}
$$

where the magnetic flux density (6) in the permanent magnet, (7) in the mixed blood stream and (8) in the human tissue and the surrounding air domain.

Combining these equations, we derive the PDE in terms of the potentials $\mathrm{A}$ and $\mathrm{V}$ :

$$
\begin{gathered}
\sigma \frac{\partial \mathrm{A}}{\partial \mathrm{t}}+\boldsymbol{\nabla} \times\left(\mu_{0}^{-1} \nabla \times \boldsymbol{A}-\boldsymbol{M}\right)-\sigma \boldsymbol{u} \times(\boldsymbol{\nabla} \times \boldsymbol{A}) \\
+\sigma \nabla \boldsymbol{V}=\boldsymbol{J}^{e}
\end{gathered}
$$

The magnetic carrier nanoparticles loaded with drug molecules and the blood in which they are mixed form a nonconducting bulk and their conductivity is neglected. Applying this hypothesis in (9) the equation is simplified to:

$$
\boldsymbol{\nabla} \times\left(\mu_{0}^{-1} \nabla \times \boldsymbol{A}-\boldsymbol{M}\right)=\boldsymbol{J}^{\boldsymbol{e}}
$$

Here $\mu 0$ is the magnetic permeability of vacuum $(4 \pi \times$ $\left.10^{-7} \mathrm{H} / \mathrm{m}\right)$; Je represents any applied external current density null in this simulation; $\mathrm{Br}$ is the remnant magnetic flux (T); and $M$ is the magnetization vector in the blood stream $(\mathrm{A} / \mathrm{m})$, which is a function of the magnetic field $\mathrm{H}$ as formulated in equation (11), and hence may be expressed in terms of $\mathrm{A}$.

$$
\mathbf{M}=\chi \mathbf{H}
$$

In this $2 \mathrm{D}$ simulation, the three fields $\mathrm{B}, \mathrm{H}$ and $\mathrm{M}$ have only two components in the $\mathrm{x}-\mathrm{y}$ plane and thus $\mathrm{A}$ will be in the $\mathrm{z}$ direction: $\mathrm{A}=(0,0, \mathrm{Az})$. The components of these vector quantities are obtained using equations (5), (7) and (11), as follows:

$$
\begin{aligned}
& \mathrm{B}_{\mathrm{x}}=\frac{\partial \mathrm{A}}{\partial \mathrm{y}} \text { and } \mathrm{B}_{\mathrm{y}}=-\frac{\partial \mathrm{A}}{\partial \mathrm{x}} \\
& \mathrm{H}_{\mathrm{x}}=\frac{1}{\mu} \frac{\partial \mathrm{A}}{\partial \mathrm{y}} \text { and } \mathrm{H}_{\mathrm{y}}=-\frac{1}{\mu} \frac{\partial \mathrm{A}}{\partial \mathrm{y}}
\end{aligned}
$$


Proc. of the Fourth International Conference on Advances in Civil, Structural and Environmental Engineering - ACSEE 2016. Copyright (C) Institute of Research Engineers and Doctors. All rights reserved.

ISBN: 978-1-63248-114-6 doi: 10.15224/ 978-1-63248-114-6-14

$$
\mathrm{M}_{\mathrm{x}}=\frac{\chi}{\mu} \frac{\partial \mathrm{A}}{\partial \mathrm{y}} \text { and } \mathrm{M}_{\mathrm{y}}=-\frac{\chi}{\mu} \frac{\partial \mathrm{A}}{\partial \mathrm{x}}
$$

Here $\chi$ represents the magnetic susceptibility of the blood mixture and $\mu$ is the magnetic permeability of the magnetized fluid. The applied values depend on the nature and the concentration of the considered ferro fluid and range from 0.1 to 0.6 [4].

\section{B. Navier-Stokes Equations:}

The dynamic representation of ferrofluid motion is based on a momentum conservation equation that assumes an incompressible Newtonian flow expressed by the NavierStokes equations [13-15]:

$$
\begin{gathered}
\rho \frac{\partial \mathbf{u}}{\partial \mathrm{t}}-\boldsymbol{\nabla} \cdot \eta\left(\boldsymbol{\nabla u}+(\boldsymbol{\nabla u})^{\mathrm{T}}\right)+\rho \mathbf{u} \cdot \boldsymbol{\nabla} \mathbf{u}+\boldsymbol{\nabla} \mathbf{p}=\mathbf{F} \\
\boldsymbol{\nabla} \cdot \mathbf{u}=0
\end{gathered}
$$

Where $\eta$ denotes the dynamic viscosity $(\mathrm{kg} /(\mathrm{m} \cdot \mathrm{s}))$, u the velocity $(\mathrm{m} / \mathrm{s}), \rho$ the fluid density $(\mathrm{kg} / \mathrm{m} 3)$, p the pressure (N/m2), and $\mathbf{F}$ the total volume force applied to the ferrofluid $(\mathrm{N} / \mathrm{m} 3)$. The hydrodynamic problem is coupled to the magneto statics problem through the magnetic body force $\mathrm{F}$ due to the fluid magnetization under the influence of the magnetic field [16].

$$
\mathbf{F}=(\mathbf{M} . \boldsymbol{\nabla}) \mathbf{H}
$$

Using Equations (13) and (14), the above relation leads to the expressions [15].

$$
\begin{aligned}
& \mathrm{F}_{\mathrm{x}}=\frac{\chi}{\mu_{0} \mu_{\mathrm{r}}^{2}}\left(\frac{\partial \mathrm{A}}{\partial \mathrm{x}} \frac{\partial^{2} \mathrm{~A}}{\partial \mathrm{x}^{2}}+\frac{\partial \mathrm{A}}{\partial \mathrm{y}} \frac{\partial^{2} \mathrm{~A}}{\partial \mathrm{x} \partial \mathrm{y}}\right) \\
& \mathrm{F}_{\mathrm{y}}=\frac{\chi}{\mu_{0} \mu_{\mathrm{r}}^{2}}\left(\frac{\partial \mathrm{A}}{\partial \mathrm{x}} \frac{\partial^{2} \mathrm{~A}}{\partial \mathrm{x} \partial \mathrm{y}}+\frac{\partial \mathrm{A}}{\partial \mathrm{y}} \frac{\partial^{2} \mathrm{~A}}{\partial \mathrm{y}^{2}}\right)
\end{aligned}
$$

Here $\mu \mathrm{r}$ is the relative magnetic permeability of the ferrofluid (dimensionless). In the present simulation, these components are multiplied by the ferrofluid mass fraction, $\mathrm{k}$ (set to be $10 \%$ of the total mass mixture) in order to get the final expression for the volume force in the blood stream [15].

\section{Boundary Conditions and sub domain parameters}

\section{A. Magneto static application}

For the magneto static part of this simulation, Dirichlet boundary conditions on the outer domain reasonably far away from the magnet are applied $\mathrm{Az}=0$. The skin, tissue and air sub domains are considered to have close magnetic properties and $\mu_{r}=1$. In the permanent magnet sub domain, the values for the remnant induction are taken from [20] and the simulation is carried out for the maximal value of $1.5 \mathrm{~T}$. Its relative permeability is chosen to be 5000 . As for the vessel canal, the presence of the nanoparticles changes the blood properties and the bulk is considered magnetized. The induced magnetization as given by equation (14) is implemented.

Once the magneto static application is solved for the vector potential A, it can be used to visualize, in post processing, the other quantities such as the magnetic field, the induced magnetic induction, the induced magnetization, the current density, the Lorentz force. .. The solution is then used as an initial condition to calculate the fluid velocity field using the Navier-Stokes equations in the time domain.

\section{B. Fluid flow application:}

Since the fluid flow takes place only in the canal, only this sub domain is active and the appropriate boundary limits and parameters are applied. For a viscous laminar streaming liquid, the flow profile is parabolic. Therefore, the inflow profile of the liquid is parabolically shaped by the normal inflow velocity. To emulate the heartbeat of 60 pulses by minute, the inflow velocity follows a sinusoidal expression in time, according to the following equation $[13,15$, and 17]:

$$
U_{0}=2 U_{\max } s(1-s)\left(\sin (\omega t)+\sqrt{\sin (\omega t)^{2}}\right)
$$

Where $\mathrm{s}$ is a boundary segment length parameter that goes from 0 to 1 along the inlet boundary segment and $U_{\max }$ is the maximal flow velocity $(50 \mathrm{~cm} / \mathrm{s})$. No-slip conditions are specified at the vessel walls. At the outlet, an outlet pressure condition, $\mathrm{p}=0$ is set to account for no shear stress [15]. To solve the Navier Stokes equations, the Ferro fluid characteristics must be implemented. In this simulation, the density of the mixture is taken from [21] to be $1060 \mathrm{~kg} / \mathrm{m} 3$ and the viscosity is chosen to correspond to $0.005 \mathrm{~Pa}$.

\section{Results and discussion}

\section{A. Magneto-statics simulation}

The solution of the magneto static problem gives information about the range of values of the magnetic flux density field in this 2D simulation which will predict the effect on the magnetized fluid flow. Figure 3 shows by streamlines, the magnetic flux lines and the markers indicate the maximum and the minimum values. In the vessel, the nanofluid far away from the magnet will be subject to weak fields of the order of $6 \times 10^{-8} T$ and closer to the magnet, the magnetic flux density will reach a maximum of $1.575 \mathrm{~T}$. This figure will be unaffected by the fluid flow since this part of the application does not depend on the hydrodynamic simulation and is time independent. It also shows that the effect of the magnetic field will be more important as we approach the extremities of the magnet and that at the entrance of the blood vessel, the field of $0.015 \mathrm{~T}$ is too weak to affect the mixture flow.

Figure 4 indicates the spatial variations in the $\mathrm{x}$-direction of the magnetic flux density at the center of the vessel. It shows the increase in magnitude as we approach the permanent magnet. 
Proc. of the Fourth International Conference on Advances in Civil, Structural and Environmental Engineering - ACSEE 2016. Copyright ( $)$ Institute of Research Engineers and Doctors. All rights reserved.

ISBN: 978-1-63248-114-6 doi: 10.15224/ 978-1-63248-114-6-14

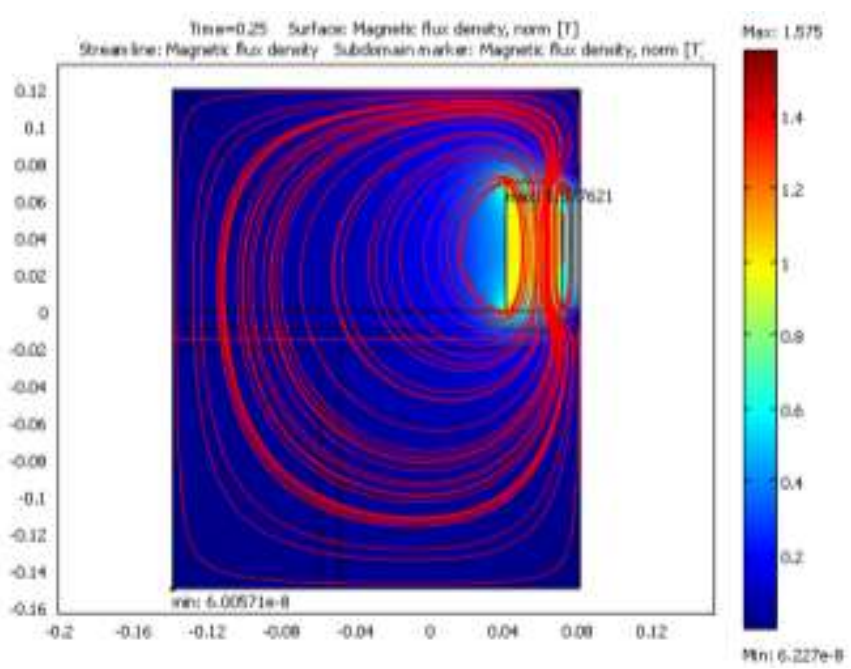

Figure 3. Magnetic flux density lines with no flow considered.

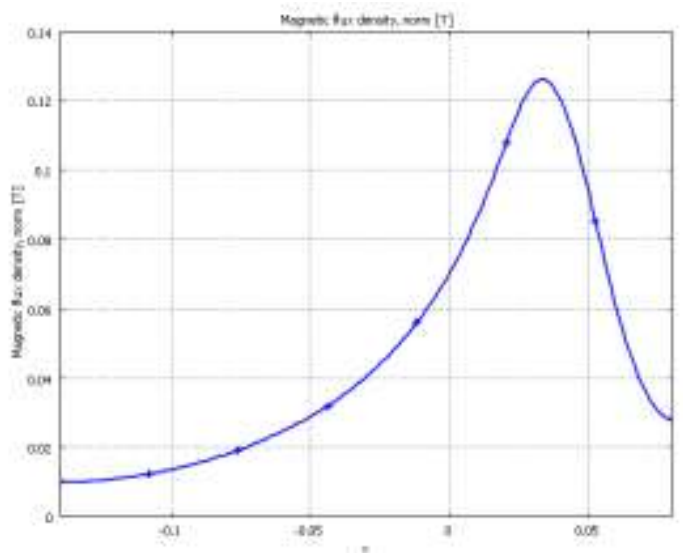

Figure 4. Field variations at the center of the vessel.

\section{B. Navier-Stokes simulation}

Once the magnetic quantities are simulated and fed into the hydrodynamics model, the solution investigates the behavior of the velocity field, the pressure,...

The following simulation indicates that after $0.25 \mathrm{~s}$, the heart beat is at its peak and the mixture velocity in the vessel reaches its maximum value of $0.50 \mathrm{~m} / \mathrm{s}$ at the entrance of the vessel as shown on figure 5 .

Due to the weak field around the first branch, we notice that, after this period, the flow seems to head for the first branch but, as time goes by, the mixture is attracted towards the desired site due to a closer stronger field which pulls the magnetized particles towards the selected location. In this phase, the ferrofluid behaves as a turbulent flow at the junction of the two branches.

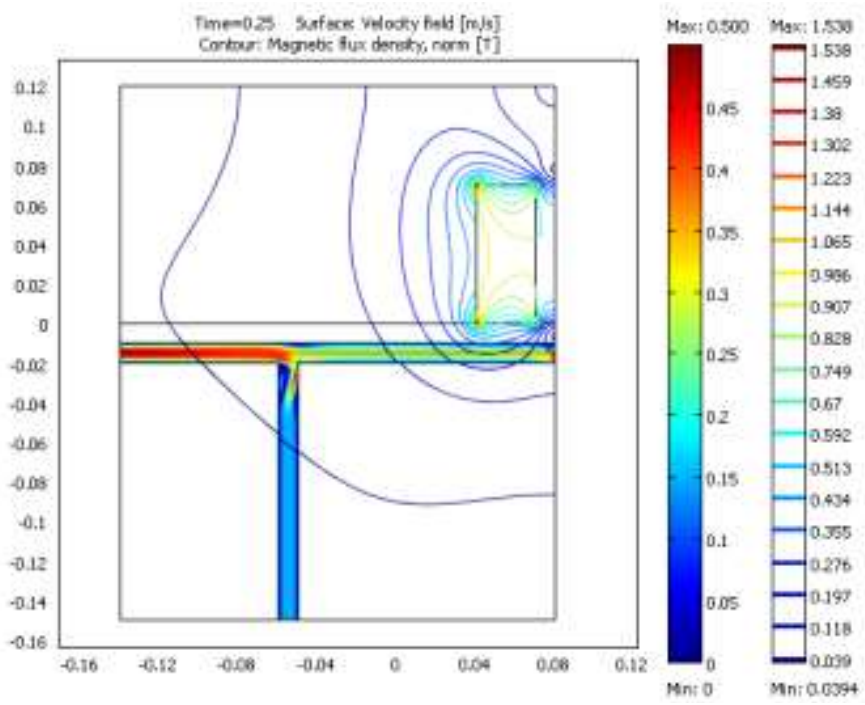

Figure 5. Velocity Field at maximum blood throughput.

To compare the different velocities with or without the influence of the magnetic field, a simulation is carried out with the incompressible NS application is removed. Results are shown in figure 6 where it is interesting to notice that the fluid reaches the end of the first branch (and exits the model) with a velocity of $0.25 \mathrm{~m} / \mathrm{s}$ instead of reaching the simulated cancer tumor.

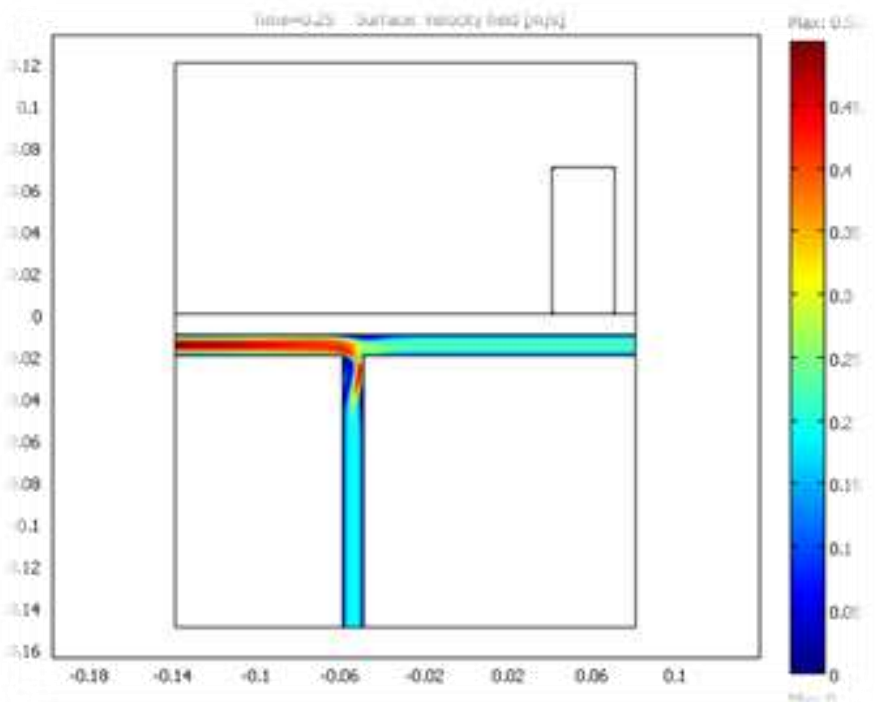

Figure 6. Velocity Field at maximum blood throughput under no magnetic field.

The velocity of the ferrofluid is simulated for different times to account for the heart beat cycle. Figure 7 shows that the maximum velocity is obtained immediately under the magnet. This figure confirms the published results [3, 4, 15] and shows that the velocity reaches its peak at the point beneath the magnet $(-0.05 ;-0.01)$ when the heart beat $(\mathrm{t}=1.25)$ is at maximum blood throughput. The influence of the magnet becomes more important as it pulls the magnetized blood more rapidly and efficiently as time goes by (the maximum velocity 
Proc. of the Fourth International Conference on Advances in Civil, Structural and Environmental Engineering - ACSEE 2016. Copyright ( $)$ Institute of Research Engineers and Doctors. All rights reserved.

ISBN: 978-1-63248-114-6 doi: 10.15224/ 978-1-63248-114-6-14

at the same point of $(-0.05 ;-0.01)$ increases from $0.31 \mathrm{~m} / \mathrm{s}$ at $\mathrm{t}=0.25 \mathrm{~s}$ to $0.33 \mathrm{~m} / \mathrm{s}$ at $\mathrm{t}=1.25 \mathrm{~s})$.

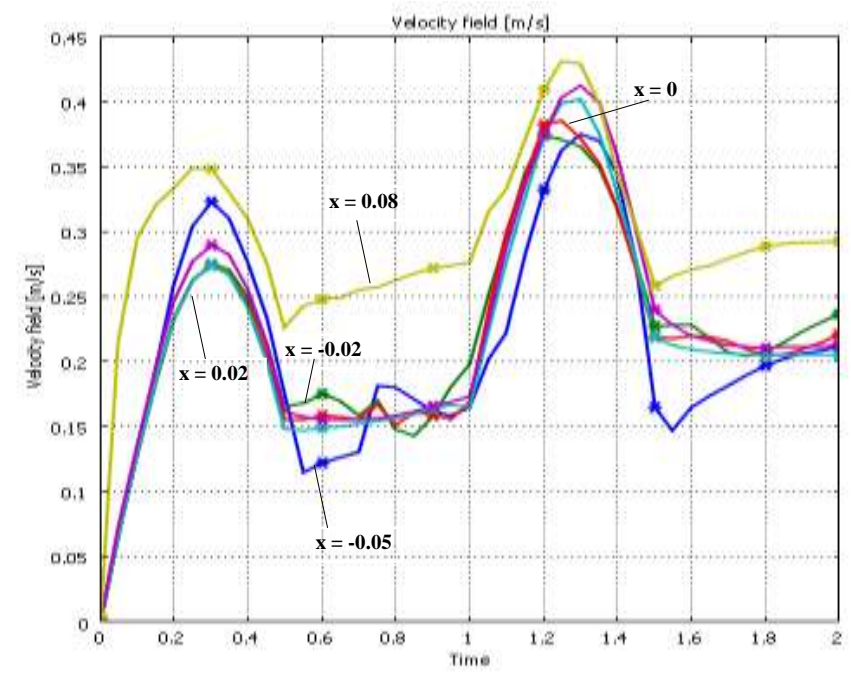

Figure 7. Velocity field along the center of the vessel in the $\mathrm{x}$ direction between two heart beats.

\section{v. Conclusion}

In this paper, a mathematical model is developed to predict the trajectories of magnetic nanoparticles in a blood vessel under the influence of a permanent magnet positioned outside the body. The simulation is carried out taking into account the heart beat cycle. The results show that the magnetic forces are able to attract the nanofluid away from a prior branch and guide it to regions beneath the permanent magnet.

\section{References:}

[1] Shashi Sharma n, V.K. Katiyar, Uaday Singh .Mathematical modelling for trajectories of magnetic nanoparticles in a blood vessel under magnetic field. Journal of Magnetism and Magnetic Materials 379 (2015) 102-107.

[2] Pierre Pouponneauab, Jean-Christophe Lerouxb,c, Gilles Soulezd, Louis Gabourye, Sylvain Martela .Co-encapsulation of magnetic nanoparticles and doxorubicin into biodegradable microcarriers for deep tissue targeting by vascular MRI navigation.Biomaterials 32 (2011) 34813486.

[3] Thodsaphon Lunnoo and Theerapong Puangmali. Capture Efficiency of Biocompatible Magnetic Nanoparticles in Arterial Flow: A Computer Simulation for Magnetic Drug Targeting. Nanoscale Research Letters201510:426 DOI: 10.1186/s11671-015-1127-5

[4] Morteza Mahmoudi, Mohammad A. Shokrgozar, Abdolreza Simchi, Mohammad Imani, Abbas S. Milani, Pieter Stroeve, Hojatollah Vali, Urs O. Häfeli, and Shahin Bonakdar. Multiphysics Flow Modeling and in Vitro Toxicity of Iron Oxide Nanoparticles Coated with Poly(vinyl alcohol), J. Phys. Chem. C2009,113,2322-2331

[5] R. Rosensweig. Ferrohydrodynamics, Chapter 9. Cambrige University Press, Cambrige, 1985

[6] Q. Cao, X. Han, L. Li, Enhancement of the efficiency of magnetic targeting for drug delivery: development and evaluation of magnet system, J. Magnetic Materials 323 (2011) 1919-1924.

[7] A. Nacev, A. Komaee, A. Sarwar, R. Probst, S.H. Kim, M.E. Buck, B. Shapiro Towards control of magnetic fluids in patients, IEEE Control Syst. Mag. 32 (2012) 32-74.

[8] B. Polyak, G. Friedman, Magnetic targeting for site-specific drug delivery: Applications and clinical potential, Expert Opin. Drug Deliv. 6 (2009) 53-70
[9] Shubayev VI1, Pisanic TR 2nd, Jin S. Magnetic nanoparticles for theragnostics. Adv Drug Deliv Rev. 2009 Jun 21;61(6):467-77. doi: 10.1016/j.addr.2009.03.007. Epub 2009 Apr 20

[10] Benjamin Shapiro, Sandip Kulkarni, Aleksander Nacev,Silvia Muro,PavelY.Stepanov and Irving N. Weinberg. Open challenges in magnetic drug targeting. WIREs Nanomed Nanobiotechnol 2014. doi: 10.1002/wnan.1311

[11] Lubbe AS, Bergemann C, Riess H, Schriever F, Reichardt P, Possinger K, Matthias M, Dörken B, Herrmann F, Gürtler R. Clinical experiences with magnetic drug targeting: a phase i study with 4-epidoxorubicin in 14 patients with advanced solid tumors. Cancer Res 1996, 56:46864693

[12] Lemke AJ, von Pilsach MIS, Lubbe A, Bergemann C, Riess H, Felix R. Mri after magnetic drug targeting in patients with advanced solid malignant tumors. EurRadiol 2004, 14:1949-1955.

[13] Gerrard, J. H. and Taylor, L. A. Mathematical model representing blood flow in arteries. Med. \& Biol. Eng. and Comput., Vol. 15,pp. 611$617,1977$.

[14] C.M. Oldenburg, S.E. Borglin, and G.J. Moridis, "Numerical Simulation of Ferrofluid Flow for Subsurface Environmental Engineering Applications," Transport in Porous Media, vol. 38, pp. 319344, 2000.

[15] Daniel J. Strauss, Mark A. Pinsky, Niklas Rom und Helmut Schmidt. Simulation von magnetischen Nano-Träger-Systemen in der Tumortherapie. (c) 2004 WILEY-VCH Verlag GmbH \& Co. KGaA, Weinheim.

[16] E.E. Tzirtzilakis. A mathematical model for blood flow in magnetic Field. PHYSICS OF FLUIDS 17, 077103. 2005

[17] Martin Brandl , Michael Mayer, Jens Hartmann , Thomas Posnicek, Christian Fabian, Dieter Falkenhagen.Theoretical analysis of ferromagnetic microparticles in streaming liquid under the influence of external magnetic forces.Journal of Magnetism and Magnetic Materials 322 (2010) 2454-2464

[18] Gaurav Varshney. Effect of magnetic field on the blood flow in artery having multiple stenosis: a numerical study. MultiCraft International Journal of Engineering, Science and Technology Vol. 2, No. 2, 2010, pp. 67-82

[19] Mallikarjuna reddy c,... mathematical model governing magnetic field effect on bio magnetic fluid flow and orientation of red blood cells Pacific-Asian Journal of Mathematics, Volume 5, No. 1, January-June 2011

[20] Aleksandar Nelson Nacev. Magnetic drug targeting: developing the basics. Doctor of Philosophy Dissertation, University of Maryland, College Park, 2013.

[21] Martin Brandl, Michael Mayer, Jens Hartmann , Thomas Posnicek, Christian Fabian, Dieter Falkenhagen. A model for predicting magnetic targeting of multifunctional particles in the microvasculature .Journal of Magnetism and Magnetic Materials 312 (2007) 187-193

About Author (s):

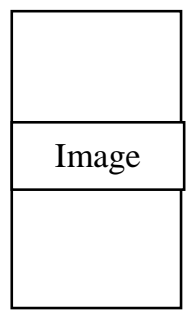

[Type a quote from the document or the summary of an interesting point. You can position the text box anywhere in the document. Use the Drawing Tools tab to change the formatting of the pull quote text box.] 